\title{
Resilient Campus Network to Support Research and Educational Network in Ghana [Case Study of Accra Technical University]
}

\author{
Felix Attuquaye Okai \\ Department of Comp.Sci \\ Accra Tech. University \\ Accra-Ghana
}

\author{
Ing. Komi Agbesi \\ Department of Comp.Sci \\ Accra Tech. University \\ Accra-Ghana
}

\author{
Victor Gbedawo \\ ICT Department \\ Accra Tech. University \\ Accra-Ghana
}

\begin{abstract}
This paper describes how to setup a fully resilient design in a campus network to support Research and Educational Network in Ghana. The main idea of resilient topology for tertiary institutional campuses is to minimize downtime to the barest possible minimum. These down times usually happen during crashes and device upgrades. This paper describes how all critical devices are used twice to avoid having a single point of failure. Therefore, any single device can be turned off without significant disruption for the connected applications and users. Finally an appropriate resilient network that will support both students and staff research and collaborative works, with adequate bandwidth requirement to render the system scalable within the next seven years has been determined and recommended.
\end{abstract}

\section{General Terms}

Resilient Campus Network, Accra Technical University

\section{Keywords}

Resilient, Campus Network, Research and Educational Network, Tertiary Institution

\section{INTRODUCTION}

Technological advancement over the past two decades has altered and spawned a new global economy that is fueled by information technology and driven by knowledge. The global trends towards a knowledge-based economy do emphasize the importance of tertiary institutions as repositories of valuable human resources to help secure shares in the global market. The current increasing shift, to a high-end-information and telecommunication driven economies, requires sustained human resource development and training [1]. (Gwang-Jo Kim, 2011). The use of information and communication technology (ICT) in education is therefore an inevitable tool for the delivery of curricula in tertiary institutions and the enabling platform that has made this possible is the Research and Education Networks (REN). It is a platform that offers both teachers and students of higher learning, the connectivity to an unlimited number of learning resources and services, relevant to their field of endeavors. The use of REN, backed by a resilient campus network (RCNET) will enable students and researchers alike, the opportunity to collaborate with each other within and across the continent.

\subsection{Problem Statement}

A policy report published by the ministry of education in Ghana on ICT in Education indicates that, for over fifteen years now, efforts towards the deployment of ICT in Tertiary Education especially in the technical universities and universities in the country have basically been focused on the provision of computers and establishment of ICT laboratories. This has been the typical case of Accra technical university (formally called Accra technical university) in the past years. This provision of infrastructure only forms a small fraction of the infrastructural needs of tertiary institutions in Ghana and particularly Accra technical university. Since the transformation of the institution into a technical university, higher demand for worldwide collaboration with academia had become inevitable. This collaboration among students and researchers in the delivery of quality research can fuel the country's economy by producing students with the requisite technological know-how to support Ghanaian industries and be able to compete with other technocrats worldwide. It is regrettable that, tertiary institutions in Ghana now, cannot boast of a resilient, stable, accessible, scalable and affordable network for the sharing of information resource among academia and students. This situation has deprived international collaboration between Ghanaian researchers, lecturers and students especially, in the institutions of higher education.

\subsection{Research Objectives}

The main objective of this paper is focused on:

a) The bottlenecks within the existing campus network infrastructure in Accra technical university, and other sister universities that hinders their full support for REN in the country (Ghana).

b) A resilient campus network infrastructure that is cost effective, stable, scalable and sustainable to connect Accra technical university to REN and to the rest of the world.

\subsection{Research and Educational Networks}

Today, high usage of the internet for teaching and learning has become the order of the day and are increasingly being adopted as a replacement to the classical way of teaching and learning in our tertiary institutions today. REN are physical high speed telecommunications network, designed to promote education and research.

REN is considered an essential infrastructure for the advancement of research and education and exist in almost every developed country. Without high-speed research network, many research projects would have simply be unavailable.

REN creates a common platform for research, fostering multinational collaboration. It is designed to be a breakthrough infrastructure within the research and education communities, providing the power to integrate many research works and teaching methodologies and forcefully driving co-operation. REN supports high speed data transfer required for advanced research and education. There is evidence that the availability of cost effective and cutting edge REN network services enables 
and encourages technological spillover into the commercial sector, which ultimately benefits society as a whole. Conversely, the absence of such facilities hampers such development and can exclude countries from achieving advances that could help their economic development [2].

\section{USER REQUIREMENT AND ANALYSIS}

Questionnaires and interviews were administered to students, academic and non-academic staffs in the institution to establish all relevant user requirements that are needed to support the most ideal access network infrastructures and bandwidth requirements that will be scalable for the next five (5) years and beyond.

\subsection{Respondents Analysis}

Responses from 198 student's questionnaires were received for analyses, representing $81.6 \%$ response rate which is a good rate for extrapolation.

The student's questionnaires were randomly distributed to one hundred and twenty (120) students across the three schools of the technical university, namely: school of applied sciences and arts, school of business studies and the school of engineering. The student questionnaires were structured to cover three broad schematic areas that include:

Interviews, with fifty (50) academic staffs spanned across their online behavior, collaborative behavior and network application services with much focus on teaching and research requirements was carried out.

\section{Interviews with Staff of I.T Department}

Four (4) senior staffs from the IT department were interviewed. The interviews were aimed at gathering enough information on the following areas;

- Existing network infrastructures.

- Network topology

- Connection to Service provider

- Network services offered.

- Existing bandwidth etc.

- Current ISP

\subsection{Analysis of Network Applications and Services}

\subsubsection{Students}

Questions grouped under these categories were aimed at finding out how much time student spent actively using data network application and services for their studies. $60 \%$ of the student indicated that they spent less than 2 hours daily using the internet, with over half of these student using network resources for less than half of the time specified above (1hour).Contrary to the above less than $10 \%$ of the students responded actively using internet for more than 4 hours daily of which majority of the students fall within the science field of studies.

From the above analysis, it is apparent that the students in the science field of study, uses the internet for their research work more frequently than those in the non-science field of studies. In effect science oriented programmes will require more bandwidth as compared to other non-science oriented students. Closed ended questions were grouped under capabilities and performance of the current campus network infrastructure in response to supporting students research and collaboration works. Almost $70 \%$ of student strongly disagreed by that accession. Questions centered on areas of whether the current network infrastructure offered continuous and reliable service and if the existing network guaranteed any security and protection of data.
From the responses gathered, it was imperative that quality of service $(\mathrm{QoS})$, data protection and network security needs to be considered more critically in the design of the CREN.

To satisfy the capabilities and network infrastructural needs of users (students, lecturers, and administrative staffs) the design of both the CNET and NREN should take into consideration a reliable and fast network offering high quality of service.

\subsubsection{Academic Staff}

The response from the academic staff was similar to that of the students' responses but with additional modification made. Most academic members presently have face-to-face interaction with fellow researchers and students. Based on this, it is required that enhancement of the current network should support much video conferencing capabilities to enhance better collaboration among fellow researchers and students worldwide. The video conference application requires about $2 \mathrm{Mbps}$. Based on the above observation a suitable research and educational network will make room for a higher bandwidth that can support video conference application.

\subsubsection{School Management}

Interviews and live data from the academic department were focused on the following category of information;

- Current student population

- Projected student population growth within 6 years.

- Student to computer ratio for the entire student population.

- Rate of introduction of new courses for the next 6 years.

- Direction of teaching and learning modes

The idea of such open ended question was to make a projection for the CNET and NREN that will serve both researches and students beyond 6 years. It was quiet clear that current lecture rooms and facilities of the institution will not be able to support growing number of students (both part time and full time) turning into the technical university. This situation requires the running of online courses to adequately address these problems. Again with the current preparation to transform the technical universities into technical universities by September, 2016, the demand for such a network facility is quite enormous.

The student population of Accra Technical university as at the end of 2015/16 academic year was twelve thousand, six hundred and eighty-five $(12,685)$. Based on figures within the past four (4) years (table 1), a projected average growth of student for the technical university within the next 5 years was determined to be approximately $5.3 \%$.

From the table below (Table 1), the design of a scalable and adaptive CNET and NREN that will support student growth rate of $5.3 \%$ was adequately designed and deployed.

Table 1: Student population

\begin{tabular}{|l|l|}
\hline Academic Year & Total Student population \\
\hline $\mathbf{2 0 1 2 - 1 3}$ & 11,773 \\
\hline $\mathbf{2 0 1 3 - 1 4}$ & 12,376 \\
\hline $\mathbf{2 0 1 4 - 1 5}$ & 11,975 \\
\hline $\mathbf{2 0 1 5 - 1 6}$ & 12,373 \\
\hline
\end{tabular}




\subsection{EXISTING CAMPUS NETWORK}

Information gathered from the interviews, revealed that A-poly has a current bandwidth of $6 \mathrm{Mbps}$. The network uses a fiber last mile connection to the internet using Vodafone as the internet service provider (ISP).

Accra Technical University currently operates a three-tier network with the core network router interface connected by fiber to Vodafone. The access network is made up of an Ethernet twisted pair star topology. The institution and its community currently has a campus wide wireless coverage with wireless Access points located at some specific locations within the technical university campus to provide wireless internet access to the technical university community.

\subsection{Vodafone Network in Ghana}

Vodafone Ghana (formerly known as Ghana Telecom), is one of the biggest Internet service providers in Ghana today, serving voice and data networks. They currently have a core network which serves as a backbone to their operations. This consists of an optical network in a three-ringed topology comprising a layer one network of an unlimited fiber. The DWDM system has a capacity of sixteen wavelengths (16 lambda) of which two are currently being used to provide a ten gigabit per second (10Gbps) core bandwidth This connects all the regional capitals to an IP/MPLS cloud sitting between layer two and three.

\section{THE PROPOSED REN DESIGN}

\subsection{Campus Network}

The campus network is mostly made up of a transmission control protocol / internet protocol (TCP/IP) network. This network has a layer three (3) device (router) which connects both the ISP on the wide area network and the campus local area network. Within the local area network an extended star topology is employed with layer two devices (switches) distributing and segmenting the network into various forms. On the wide area network, there exist a point to point connection between the ISP and the campus. A campus may decide to add additional connectivity based on their needs.

From the design point of view campus networks will be managed by the individual institutions with available help from the research and educational network to ensure efficient use of the network.

\subsection{The Access Network}

The access network is basically controlled by the ISP and acts as a path for the campus network to communicate externally. Various connectivity options and media exist based on the need of the campus. Depending on the location and demand of the campus, fiber, microwave, or copper links could be extended from the ISP's point of presence (PoP) to the campus. A combination of these technologies could be bundled together to the campus to prevent any single point of failure within the network.

In Ghana, most of the ISP's (Vodafone, MTN, Tigo, Airtel, etc) currently provide data services mostly using microwave technology with a few using fiber. Due to the reliability, speed and amount of data passing through the REN, an access network using fiber, i.e Vodafone's network is recommended.

\subsection{The Core Network}

The core network describes how the ISP routes packets (data, voice and video) from one point of the country to the other. This setup can be done in several modes using Ethernet, microwave or fiber as the transmission medium in layer one of the OSI model and technologies such as SDH/SONET and IP. Various multiplexing techniques such as time division multiplexing (TDM), wave division multiplexing (WDM) etc are employed to achieve the bandwidth required for the core of the network. The national backbone currently comprises of a dense wave division multiplexing technology which connects all the regional capitals together with a bandwidth of ten gigabits per second (10Gbps) and further connects the metro network for each region via a gigabit passive optical network. The new design leverages on this technology to design our REN.

\subsection{International Connectivity}

The international connectivity options deals with how the nation is able to connect to the rest of the world. Currently there are two main ways of achieving this in Ghana. One is by satellite and the other by an undersea fiber cable. Three companies currently have landed their cables on our shores but two are very operational.

SAT3/WASC (Southern Africa - Western Africa Submarine cable) is a $15000 \mathrm{~km}$ high-performance fiber optic cable linking Europe with South Africa and a number of countries on the West African coastline. [7] provides a capacity of 130 gigabits per second (Gbps),.

Main One Cable System is a 7,000km submarine cable which links Europe to Africa with landing points in Nigeria, Ghana and Portugal. The Cable delivers high speed bandwidth of 1.92 terabits per second (Tbps), (MainOne).

Glo1and WACS are other sources for international connectivity. Although MainOne currently has a bigger bandwidth, it has fewer landing sites than SAT3 hence we would use the SAT3 backbone for our international connectivity whiles relying on the MainOne network for backup Figure 5 below shows the landing sites of the international connectivity along the shores of Africa

\section{TECHNICAL DESIGN OF THE PROPOSED REN TO SUPPORT ACCRA TECHNICAL UNIVERSITY NETWORK}

The design goals were based on scalability, resilience, affordability, reliability, manageability and performance. Based on the user requirements, questionnaires and analysis, we came out with a table of requirements below which would serve as a basis for the design. ATU would serve as a national node which would house the equipment for the REN due to its robust and efficient design infrastructure.

Table 2: Summary of User Requirement Analysis

\begin{tabular}{|c|c|}
\hline \multicolumn{2}{|c|}{ Summary of User Requirement Analysis } \\
\hline Summary of Analysis & $\underline{\text { ATl }}$ \\
\hline CurrentStudent Population & 12 \\
\hline Avalaldble Bandwidth For Institutions & $6 \mathrm{Ml}$ \\
\hline Average Utilization & $4.9 \mathrm{~N}$ \\
\hline Perentage of usage of Bandwidth Intensive Applications & \\
\hline Access Infrastructurure to Institutions & \\
\hline Less Bandwidth Intensive Applications & \\
\hline
\end{tabular}

From table 3 above, it could be deduced that we fiber on the core backbone of Vodafone can be leased since currently Vodafone is using only two wavelengths on their DWDM core network which has sixteen wavelengths. This would provide a dedicated channel on which the REN would be run so as to connect the institution and all subsequent subscriptions from other technical universities and universities. 


\subsection{Bandwidth per user Statistics}

The bandwidth per user statistics can be obtained from the formula below bandwidthperuser $(B)$

$$
\begin{aligned}
= & \text { AvailableBandwidth }(B a) \\
& / \text { TotalPopulation }(P t) \\
B= & \frac{B a}{P t}(\text { bpspu })
\end{aligned}
$$

Where bpspu is bits per second per user

ATU;

$$
B=\frac{(6 * 1024 * 1024)}{12685}=\frac{6291456}{12685}=495.97 \mathrm{bpspu}
$$

This signifies that currently ATU has a user to bandwidth ratio of $1: 4175 \mathrm{bps}$.

$$
B a=B * P t
$$

$$
B a=1024 * 1024 * 29754=31199330304 \text { bps }
$$

Converting to gigabytes would result in

$$
B a=\frac{31199330304}{1024 * 1024 * 1024 * 8}=3.63 \mathrm{GBps}
$$

For a projected user to bandwidth ratio of $1: 1 \mathrm{Mbps}$ we would need

$$
B a=1024 * 1024 * 12685=13301186560 b p s
$$

Equivalent to

$$
B a=\frac{13301186560}{1024 * 1024 * 8}=1586 \text { MBps }
$$

For a projected growth rate of five percent per institution for five years, the bandwidth requirement for ATU becomes

$$
\begin{gathered}
B a=((1586 M B p s) * 0.05)+1586 M b p s \\
=1665 M B p s
\end{gathered}
$$

From this analogy, eight gigabit( $8 \mathrm{~Gb})$ pipe from Vodafone's core network can be conveniently leased to provide the infrastructure on which the institution would be run.

\subsection{The proposed REN design for the Technical University}

The institution will connect to the internet via an internet service provider (Vodafone). This model limits all the traffic from the institution to be directed through the internet.

To ensure a better network model, the design makes it possible for individual institutions that may later come on board to route research specific queries through the REN while general queries will be routed through the internet gateway.

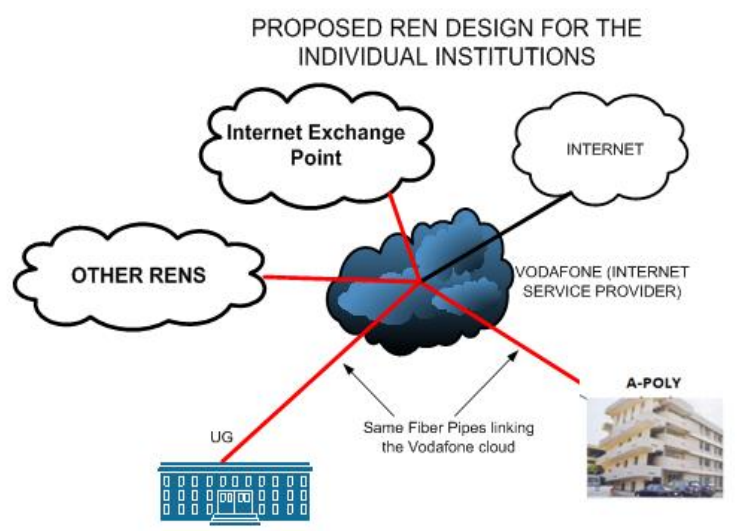

Figure 1: Proposed REN design for Accra technical University

\subsection{CAMPUS NETWORK DESIGN}

The main focus of the design activity is on the Campus Network since it is the foundation for all Research and Education activity and without a good campus network, the Research and Education Network can't work as expected. Ad-hoc campus networks usually works fine but tend to fail during demand for high speed external links. They are also difficult to maintain as the network grows.

As a result a modular and hierarchical network design was adopted with a clear separation of functions:

i. Core: Resilient, few changes, few features, high link and high CPU capacity

ii. Distribution: Aggregation, redundancy

iii. Access: Port density, affordability, security features, many adds, moves and changes.

\subsubsection{Typical Core Network}

At the core of the network are routers where data packets are routed and not switched to enhance subnet isolation.

\subsection{The Resilient Network Design}

In computer networking: resilience is the ability to provide and maintain an acceptable level of service in the face of faults and challenges to normal operation. Threats and challenges for services can range from simple misconfiguration over large scale natural disasters to targeted attacks. In order to increase the resilience of a given communication network, the probable challenges and risks have to be identified and appropriate resilience metrics have to be defined for the service to be protected, [4].

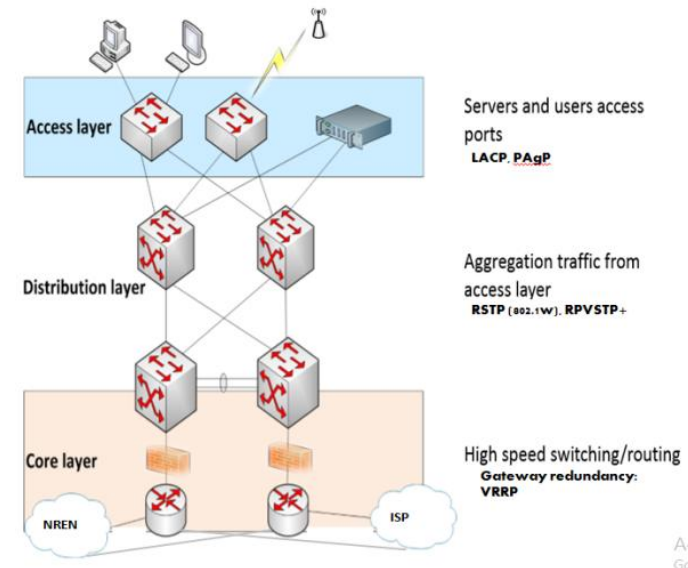

Fig 2: The Proposed Resilient Network.

The resilient Network topology will be divided into several layers. Each layer will use a different set of protocols and will require different configuration options. The following layers are used for the Resilient Campus Network:

\subsubsection{Access Layer}

This layer is used to connect end devices (servers, user's computers, printers, etc). It is strongly proposed that servers should be connected via two network adapters. For other devices which do not support resilient network connections it must be terminated on the last switch. For almost all devices which are connected through a single Ethernet port, special protocols are not required.

A slightly different situation is with a server connection where we would like to connect each one using two independent Ethernet ports. 


\subsubsection{Server access layer redundancy}

For true resiliency, the servers will have a controlled redundant network connections. This can be achieved using

i) Link aggregation protocol (LACP, PAgP), (ii) Server virtualization (iii) Ethernet card bonding (iv) Service load balancing

Link aggregation Allows combination of several physical links to form one logical channel [9]. Two signaling protocols exist for Link aggregation protocol. Thus PAgP and LACP. However LACP - IEEE 802.3ad is preferred, to minimize cost, but again, $\mathrm{PAgP}$ which is Cisco proprietary software can also be purchased for the project.

Again, static configuration (mode on) should be used since dynamic configuration usually delays channel establishment.

\subsubsection{The Distribution Layer}

This layer primarily provides L2 distribution through the switched network. This distribution layer contains redundancies which need to be eliminated and it can be resolved by using any L2 loop-free protocol. Among the most widely used protocol for this solution is the Spanning Tree Protocol (STP). However to realize our objectives in this work, the RSTP $(802.1 \mathrm{w})$ is recommended. This recommendation is based on the fact, that RSTP (802.1w) has the fasters convergence time of less than one (1) seconds and backwardly compatible with IEEE 802.1D. The topology of the distribution layer is designed to ensure that there is no ring containing more access switches. The access switch is connected to their core switch by two independent uplinks. This connectivity allows the creation of a more transparent topology and minimizes the number of VLANs on the access switches.

\subsubsection{Core Layer}

Core switches and core routers are placed in the core layer. This ensures L3 availability of the gateway IP address when one of the routers goes down. In this design the routing and switching capability has been integrated into one device.

\subsubsection{The Core Layer Components}

The VRRP protocol (RFC 3768) is used to backup the subnet gateway address. In VRRP there is a Master and a Backup router. The router which acts as Owner, periodically sends advertisement packets to the network and the Backup router listens to them. If the Backup router does not receive three consecutive advertisement packets from the Owner, then it assumes that of the gateway address and acts as the Owner router until a new advertisement packet is received. Therefore, the current active gateway router is known as the Owner, and this can apply to either the Master or the Backup router, depending on whether the Master router is functioning properly.

\subsubsection{Backbone Layer}

The Backbone layer is a common part of the routed network. To distribute routing information a dynamic routing protocol (e.g. OSPF, RIP, BGP) is used. The routing protocols support the mechanism of resilience and topology changes by default.

\section{RECOMMENDATION AND CONCLUSION}

\subsection{Recommendations}

To ensure sustainability and deployment of the designed REN described in this paper, the following points should be adhered to;

All tertiary institutions, such as Accra technical university and any other institution that will later connect to the network should be involved in the management and payment of their operational cost at their campus level.

To have a true representation of REN in Ghana, other institution of higher learning should be brought on board to connect to the local REN.

For effective and efficient operation of REN, Collaboration between Ministry of Education, Ministry of Communications, ATU, and other sister institutions are needed to commit initial funds into the project.

\section{CONCLUSION}

In this age of technological advancement, REN is an essential tool in all institutions of higher learning. The development of REN however requires a well-resourced network infrastructure and financial support. This has been the case in well advanced parts of the world. However a resilient campus network forms the bedrock for every successful REN. Therefore it is prudent that one should always focus on the campus network first.

Ghana today has seen several initiatives to develop REN to interconnect the tertiary institutions and other research centers.

The National fiber optic backbone, Telecom networks, the SAT-3 undersea cable, Globacom undersea cable and the MainOne cable, as well as campus-wide networks at various educational institutions are good candidates for these projects.

With fiber cable becoming more and more accessible throughout the country and other international fiber links being available in Ghana such as SAT-3, MAINONE and GLO, implementing the technologies described in this project with the appropriating supporting resilient campus networks as well as a good ownership and funding models will produce a good and sustainable REN for Ghana.

\section{REFERENCES}

[1] Gwang-Jo Kim, (2011). ICT for higher education, Case study from Asia and the Pacific. http://unesdoc.unesco.org/images/0021/002141/214143E.p $\mathrm{df}$

[2] John Dyer, (January, 2009). The Case for National Research and Educational Networks (NRENs. https://www.terena.org/publications/files/20090127-casefor-nrens.pdf, Page 6

[3] Dr Donald R. Riley, (April 2011). The Limits and Barriers to Data Sharing. Artificial barriers to data sharing Technical aspects presented at The International Symposium on The Case for International Sharing of Scientific Data: A focus on developing countries.

[4] Smith, P. et al., (July, 2011) "Network resilience: a systematic approach", IEEE Communications Magazine. vol.49, no.7, pp.88-97,DOI:10.1109/MCOM.2011.5936160

[5] The Resume Net Project - The need for resilience in current and future networks

[6] MainOne, http://www.mainonecable.com/networkpage.php

[7] Safe-SAT3. http://www.safe-sat3.co.za/

[8] National Research and Education Networks in Africa Report. Pdf, Pages $10-11$

[9] Link Aggregation http://www.freebsd.org/doc/en/books/handbook/networkaggregation.html , Link aggregation in FreeBSD

[10] UNESCO. (2009). ICTs for higher education. Paper presented at the 2009 World Conference on Higher Education (WCHE), Paris. 